Article

\title{
Wireless Power and Data Transmission System of Submarine Cable-Inspecting Robot Fish and Its Time-Sharing Multiplexing Method
}

\author{
Guodong Chen ${ }^{1,2}$, Yue Sun ${ }^{1, *}$, Junxin Huang ${ }^{1}$, Boda Zhou ${ }^{2}$, Fanchao Meng ${ }^{1}$ and \\ Chunsen Tang 1 \\ 1 College of Automation, Chongqing University, Chongqing 400044, China \\ 2 Zhoushan Electric Power Company, Zhoushan 316000, China \\ * Correspondence: syue@cqu.edu.cn; Tel.: +86-135-0836-8896
}

Received: 25 June 2019; Accepted: 25 July 2019; Published: 26 July 2019

\begin{abstract}
In this paper, a hybrid system topology with one-way wireless charging function and the function of the bi-directional data communication is proposed for the problem of electric energy replenishment and data transmission faced by robot fish in the implementation of autonomous submarine cable inspection. Three working modes of the system and the time-sharing multiplexing method are studied. In the power transmission mode, high-efficiency wireless charging is realized by utilizing the transmission characteristics of a series-series (SS)-type resonant network which involves series resonant networks in both the primary side and the secondary side. In the alignment detection and handshake communication mode, the charging platform distance recognition and the handshake signal transmission are implemented through a series-parallel (SP)-type resonant network based on the ASK (amplitude shift keying) modulation method. In the high-speed data transmission mode, the reverse (secondary to primary) high-speed transmission of the inspection data is achieved through a SP-type resonant network based on the OFDM (orthogonal frequency division multiplexing) modulation method. The three modes share the same coupled coils via a reconfigurable resonant network. The working principle of the system is expounded, the system characteristics under each working mode are analyzed, and the time-division multiplexing control strategy is given. The rationality and effectiveness of the scheme are verified by experiments.
\end{abstract}

Keywords: wireless power transmission; shared channel; energy and signal time-sharing transmission; bi-directional data communication

\section{Introduction}

With the further development of the 21st Century Maritime Silk Road, the marine economy and energy interconnection have become of key interest, which puts higher demands on energy security and power supply reliability. Compared with the mainland power supply, the special coastal environment of the coast makes the overhead lines subject to many restrictions, as a result, the submarine cable becomes the first choice for island power supply. Due to the special and complex marine environment, the safety operation and maintenance of submarine cables confronts great challenges. Due to the special and complex nature of the marine environment, the safety operation and maintenance of submarine cables is facing severe challenges. Setting warning signs, submarine cable monitoring and alarming, and improving the submarine cable manufacturing process are the main means of strengthening the protection of submarine cables. However, these methods are limited to monitoring and protection in areas above the sea surface. It is impossible to monitor the complex seabed conditions where the submarine cables are located [1]. Aiming at this practical problem, an ideal solution is taking use of underwater robots to monitor the submarine cable and the seabed environment to achieve the 
protection of the submarine cable. In recent years, research on cable-inspecting robot fish has made great progress [2], However, presently the power supply and data transmission of the underwater robot system is achieved by getting the robot fish out of water and electrically connecting it to the inspection ship, and the flexibility and safety thereof are still greatly limited. Therefore, in order to adapt to the complex seabed environment, the robotic fish cable-inspection system that can run autonomously without the inspection ship will have better practical value and application prospects [3].

For battery-powered submarine cable-patrol robot fish, the replenishment of electrical energy and the transmission of inspection data are important. For the problem of electric energy replenishment, the traditional solution is to manually charge the robot when it comes up to the water surface after patrol, but this method has low timeliness and poor operability [4]. In recent years, the rise of wireless power transmission technology has provided an ideal solution for the charging [5-7]. Underwater wireless power transmission technology can transfer electrical energy to load devices in a non-electrical contact way. In this transmission mode, there is no electrical connection between the power transmitting end and the power receiving end, which solves the problems of electric leakage and short circuit caused by the traditional plugging and unplugging mode used under water, and achieves the safety and flexibility of the underwater device power supply [8].

In terms of wireless charging of robot fish, there are few related researches at home and abroad. For the seabed environment mentioned in this paper and the wireless charging of robotic fish with high-speed data transmission requirements, the existing research cannot meet the demand [9]. In respect of signal transmission, a lot of work have been done on wireless power and signal transmission. Literature $[10,11]$ proposed a scheme for parallel transmission of energy and signals in an ECPT (electric-field coupled power transmission) system. Specifically, literature [10] proposed the parallel transmission of energy and signal by adding additional signal transmission channels. Literature [11] presented a parallel transmission method of energy and signal based on square wave carrier duty cycle modulation, which loads the signal into the energy channel for parallel transmission. Literature [12,13] proposed a parallel transmission scheme of energy and signal in an ICPT (inductively coupled power transfer) system, and introduced a scheme of modulating digital signals and then loading them onto energy coils for simultaneous transmission. In the seawater environment, data transmission is usually achieved by acoustic method [14,15]. In the submarine cable-inspection system, due to the high salinity seawater environment and the need to deal with two kinds of signals, which are the handshake communication signals between the robot fish and the charging platform, and a large amount of detection data collected by the robot fish, the solutions mentioned in the existing literature cannot meet this special demand regarding energy and data transmission.

Therefore, this paper proposes a wireless energy and data transmission composite topology based on magnetic coupling resonance technology for the problem of wireless charging and data transmission of the submarine cable-inspection robot fish. Through the time-sharing transmission of electric energy and data, the transmission processes of electric energy and data are not affected by each other, thereby effectively improving the stability and reliability of the system working process. In this paper, the system characteristics of this composite topology working in three modes are studied. The time-sharing multiplexing method for energy and signal wireless transmission and its mode switching strategy are proposed, which solves the problem of underwater wireless charging and communication of submarine cable-inspection robot fish.

\section{Energy and Data Time-Sharing Transmission Scheme}

The working scene of the robot fish based on the method described in the introduction is shown in Figure 1. 


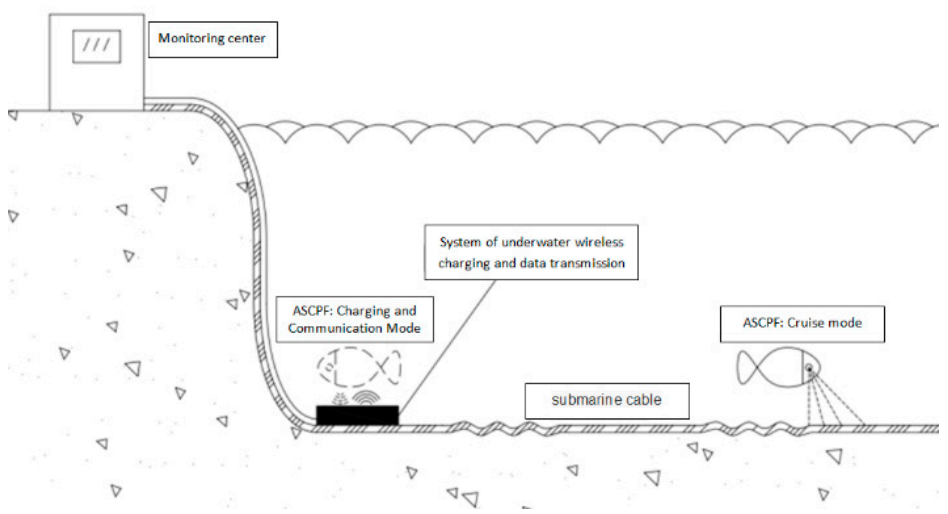

Figure 1. Cable-inspection robot fish working scene with wireless charging and data transmission.

It is docked on the platform of underwater wireless charging and data transmission after inspection of robot fish. The platform identifies the load by the resonant coil current magnitude, and sends a handshake command after identifying the load. The robot fish responds to the command and sends battery status information to the platform. After the platform and the robot fish shake hands successfully, the platform wirelessly charges them and the robot fish transmits the inspection data to the monitoring center on the shore through the platform after the charging is completed.

According to the wireless charging requirements of sea cable-inspection robot fish and its forward and reverse data transmission requirements, the structure of the energy and signal time division transmission system, as shown in Figure 2, is proposed. As we can see in Figure 2, the main topology consists of a BUCK converter, a full bridge inverter, a coupled reconfigurable resonant network, a rectifier, and the load. The BUCK converter regulates the transferred power by adjusting the input voltage of the inverter. The inverter converts the dc voltage into high frequency square wave ac voltage and stimulates wireless power transmission via the coupled resonant network. Then the rectifier converts the induced ac voltage into dc voltage and output to power the load. The coupled reconfigurable resonant network consists of a series-series (SS)-type network for power transfer (labeled by the red lines in Figure 2), a series-parallel (SP)-type for distance detection and handshake communication (labeled by the green lines in Figure 2), a SP-type network for high speed signal transmitting (labeled by the blue lines in Figure 2), and the three networks can be configured by the switches $\mathrm{S}_{5}$ and $\mathrm{S}_{6}$.

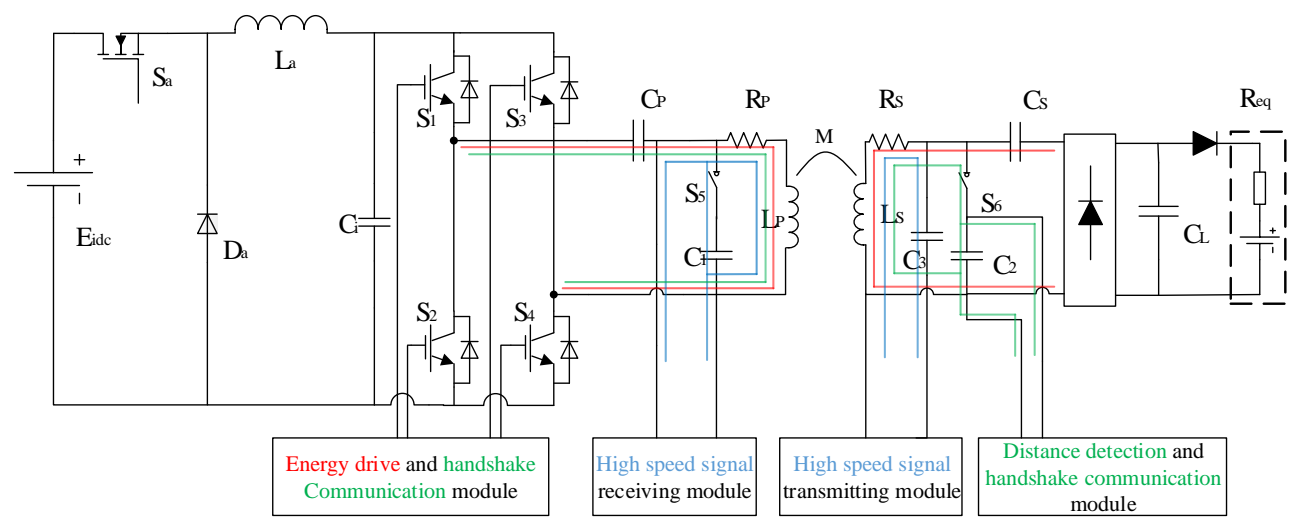

Figure 2. Energy and data time-sharing transmission system structure diagram.

In the scheme shown in Figure 2, there are three modes of operation:

Mode 1 is the energy transmission mode, which is to achieve the wireless charging function of the charging platform to the robot fish. In this mode, the main circuit topology of the system is: BUCK + full bridge inverter + SS (primary side series compensation, secondary side series compensation) resonant network + rectifier + load (robot fish battery). The reconfigurable resonant network is configured as 
SS-type by turning off $S_{5}$ and $S_{6}$. Battery charging power is regulated by adjusting the duty cycle of the BUCK link switch $S_{a}$. The switching frequency of the full-bridge inverter link is the same as the resonant frequency $f_{0}$ of the SS resonant network, which can reduce switching losses and reduce EMI (electromagnetic interference) $[16,17]$ in the soft-switching operation mode of the inverter switches.

Mode 2 is an alignment detection and handshake communication mode, which is to complete the transmission and reception of the handshake signal from the charging platform to the robot fish, and the robot fish calculates the distance to the platform by recognizing the signal strength. The reconfigurable resonant network is configured as SP-type by turning off $\mathrm{S}_{5}$ and turning on $\mathrm{S}_{6}$. This mode uses ASK (amplitude shift keying) modulation to transmit data. The primary-to-secondary signal transmission modulates the digital signal with the inverter bridge control frequency $f_{1}\left(f_{1}>>f_{0}\right)$, because the control frequency of the inverter bridge is much larger than the resonant soft switching frequency $f_{0}$ of the resonant network, the system operates in a non-resonant state. Therefore, the transmission power is greatly reduced with a large loop impedance and achieves low-power data transmission. Because the resonant frequency $\left(f_{0}\right)$ of secondary side's power transmission channel is much lower than the primary modulation frequency $\left(f_{1}\right)$, the pickup voltage will not be able to charge the battery after rectification and filtering, so that the series resonant circuit is in an open state and does not consume power. When the switch $S_{6}$ of the signal receiving module is closed, the capacitor $C_{2}$ and the inductance $L_{S}$ of the pickup coil constitute a parallel resonant circuit, whose frequency is close to $f_{1}$, so that a high signal voltage can be picked up. During the process of the robot fish approaching the charging platform, the amplitude of the picked-up signal gradually increases, and the robot fish can determine the offset distance to the platform based on the amplitude of the picked-up signal. When the amplitude of the picked-up signal reaches the maximum value, it indicates that the robot fish is directly above the charging platform, and then the robot fish can be controlled to park at the point. At the same time, the voltage can be demodulated by the signal demodulation circuit to demodulate the handshake signal sent by the charging platform. The robot fish can transmit a feedback signal to the platform by controlling the switch $S_{6}$ to be on or off, and the platform demodulates the handshake signal from the envelope of the output current.

Mode 3 is a high-speed signal transmission mode, which is to complete the inspection data transmission from the robot fish to the charging platform. The reconfigurable resonant network is configured as SP-type by turning on $\mathrm{S}_{5}$ and turning off $\mathrm{S}_{6}$. Because the amount of inspection data is large, it is designed as a high-speed signal transmission channel based on OFDM (orthogonal frequency division multiplexing) mode. The data to be transmitted is loaded into the SP-type resonant transmission network composed of $L_{S}, C_{3}, L_{P}$, and $C_{1}$ through the signal modulation transmitting module. The signal secondary side demodulates the transmitted inspection data from the capacitance voltage signal of the P-type (parallel-type) pickup network.

As described above, the three working modes share the same coupled transmitting and receiving coils $L_{p}$ and $L_{s}$. The system runs among the three working modes through a time-sharing multiplexing method to achieve accurate parking, high-efficiency wireless charging, and high-speed data transmission. Next, the characteristics of the three working modes will be analyzed and then the time-sharing multiplexing method for mode switching will be given.

\section{Analysis of the Three Working Modes}

\subsection{Characteristics of Energy Transmission Mode}

In the energy transfer mode, the equivalent circuit of the system is shown in Figure 3. The energy wireless transmission is realized by the SS resonance network, and the received electric energy is rectified and filtered, and then output to the battery for charging, and the input voltage is adjusted through the BUCK link. 


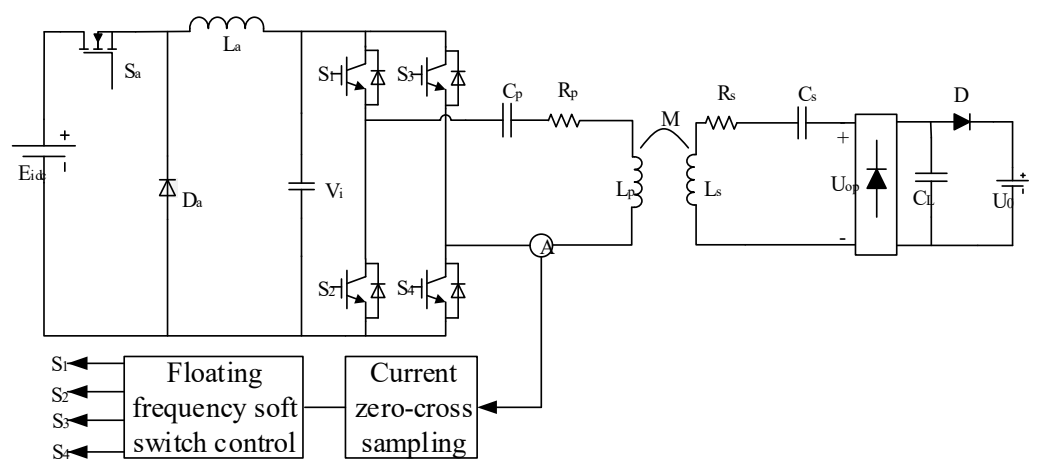

Figure 3. Energy transmission topology.

The voltage equation of the secondary resonant circuit can be obtained as follows:

$$
\mathrm{j} \omega \mathrm{MI}_{p}=\left(j \omega L_{s}+\frac{1}{j \omega C_{s}}+R_{s}\right) I_{s}+U_{o p}
$$

In the continuous state of charge of the current, the battery charging voltage can be approximated as follows:

$$
U_{o}=\left|U_{o p}\right|
$$

When the operating frequency is consistent with the secondary side resonant frequency, the following Equation (3) can be established:

$$
\mathrm{j} \omega \mathrm{M} I_{p}=R_{s} I_{S}+U_{o p}
$$

During the charging process, the battery voltage rises slowly and steadily. Therefore, it can be known from the above equation that the primary side resonant current $I_{P}$ also follows the battery voltage slowly changing. In the case of ignoring the secondary side internal resistance, the magnitude of $I_{P}$ can be approximated by the following formula:

$$
I_{p}=\frac{U_{o p}}{\omega M}
$$

The primary resonant loop voltage can be expressed by Equation (5).

$$
U_{p}=\left(j \omega L_{p}+\frac{1}{j \omega C_{p}}+R_{p}\right) I_{p}-j \omega \mathrm{MI}_{s}
$$

When the operating frequency is consistent with the primary side resonant frequency and the primary internal resistance $R_{P}$ is ignored, it is known from Equation (5) that the amplitude of the secondary current $I_{S}$ is approximately determined by Equation (6):

$$
I_{s}=\frac{U_{p}}{\omega M}
$$

Therefore, from Equations (4) and (6), the topology in which the primary and secondary sides are series resonant is used. In battery charging, the system main circuit topology has the following energy transmission characteristics:

The amplitude of the primary side resonant current $I_{P}$ is approximately determined by the output voltage $U_{o p}$, and the charging power is determined by the input voltage $U_{P}$. When designing the 
parameters, it is necessary to meet the same resonance parameters of the primary and secondary sides, as follows:

$$
\omega_{0}=\frac{1}{\sqrt{L_{p} C_{p}}}=\frac{1}{\sqrt{L_{s} C_{s}}}
$$

\subsection{Characteristics of Alignment Detection and Handshake Communication Mode}

The equivalent topology of the handshake communication signal transmission is shown in Figure 4. When the handshake communication signal is transmitted, the secondary side switch $\mathrm{S}_{6}$ is closed, so that $C_{2}$ is connected in parallel with the resonant coil. The primary side modulates the signal in the ASK mode, and the signal received by the secondary side is reflected as the amplitude change of the $\mathrm{C}_{2}$ terminal voltage, and the control command signal can be obtained by demodulating the voltage characteristic.

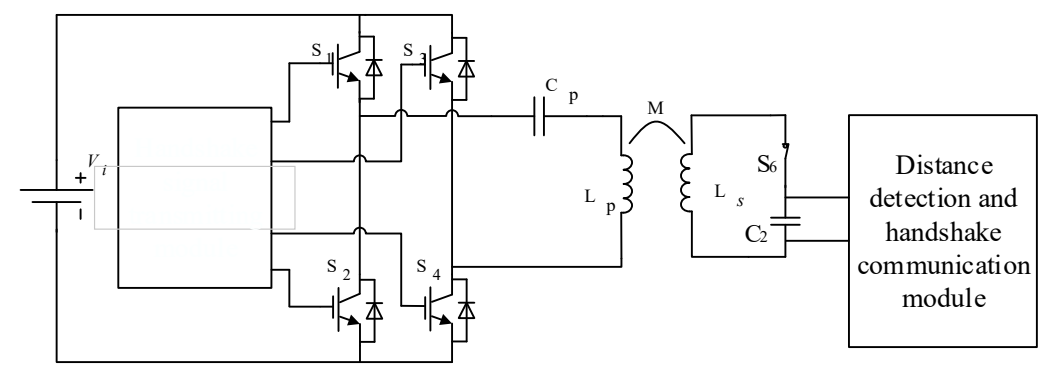

Figure 4. Low-speed signal transmission equivalent topology.

It can be seen from Figure 4 that when the handshake communication signal is transmitted, the resonant network topology is equivalent to the SP topology. The total impedance of the secondary side resonant circuit can be expressed as Equation (8):

$$
Z_{s}=j \omega L_{s}+\frac{1}{j \omega C_{s}+\frac{1}{R_{L}}}
$$

where $R_{L}$ is the input impedance of the positive signal receiving module, and its resistance is large.

According to Ohm's law, the resonant current of the pick-up loop can be calculated at this time.

$$
I_{s}=\frac{U_{s}}{Z_{s}}=\frac{j \omega \mathrm{M} I_{p}}{Z_{s}}
$$

where $I_{\mathrm{P}}$ is the primary side transmitting coil current, and when the resonant network input voltage is $U_{\mathrm{P}}$, the primary side current $I_{\mathrm{P}}$ is represented by Equation (10).

$$
I_{s}=\frac{U_{s}}{Z_{s}}=\frac{j \omega \mathrm{M} I_{p}}{Z_{s}}
$$

The equivalent impedance $Z_{\mathrm{P}}$ is established by the following equation:

$$
I_{s}=\frac{U_{s}}{Z_{s}}=\frac{j \omega \mathrm{MI}_{p}}{Z_{s}}
$$

The parallel Equations (9) and (10) can obtain the secondary side resonance voltage, that is, the input voltage of the handshake communication signal receiving module is expressed as Equation (12):

$$
U_{R_{L}}=I_{s} \frac{1}{j \omega C_{s}+\frac{1}{R_{L}}}=j \omega M \frac{U_{p}}{Z_{p} Z_{s}+\omega^{2} M^{2}} \frac{1}{j \omega C_{s}+\frac{1}{R_{L}}}
$$


From Equation (12), the voltage gain of the signal transmission channel is established by Equation (13):

$$
U_{R_{L}}=I_{s} \frac{1}{j \omega C_{s}+\frac{1}{R_{L}}}=j \omega M \frac{U_{p}}{Z_{p} Z_{s}+\omega^{2} M^{2}} \frac{1}{j \omega C_{s}+\frac{1}{R_{L}}}
$$

The frequency response function of the forward data transmission channel is obtained by the following equation:

$$
U_{R_{L}}=I_{s} \frac{1}{j \omega C_{s}+\frac{1}{R_{L}}}=j \omega M \frac{U_{p}}{Z_{p} Z_{s}+\omega^{2} M^{2}} \frac{1}{j \omega C_{s}+\frac{1}{R_{L}}}
$$

Under the parameters shown in Table 1, the frequency response characteristic curve is shown in Figure 5 below. According to the frequency response characteristic curve, $200 \mathrm{kHz}$ with a gain of about $-37 \mathrm{~dB}$ is selected as the communication frequency, which not only ensures the signal strength, but also makes the power consumption reach a relatively low level.

Table 1. System parameter table.

\begin{tabular}{cc}
\hline Parameter Name & Parameter Value \\
\hline DC input voltage, $\mathrm{E}_{\mathrm{dc}}$ & $48 \mathrm{~V}$ \\
Primary resonant inductance, $\mathrm{L}_{\mathrm{P}}$ & $257 \mathrm{uH}$ \\
Primary resonant capacitance, $\mathrm{C}_{\mathrm{P}}$ & $13.64 \mathrm{nF}$ \\
Primary coil internal resistance, $\mathrm{R}_{\mathrm{P}}$ & $0.2 \Omega$ \\
Mutual inductance, $\mathrm{M}$ & $33.1 \mathrm{uH}$ \\
Secondary resonant inductance, $\mathrm{L}_{\mathrm{S}}$ & $257 \mathrm{uH}$ \\
Primary resonant capacitance, $\mathrm{C}_{\mathrm{S}}$ & $13.64 \mathrm{nF}$ \\
Secondary coil internal resistance, $\mathrm{R}_{\mathrm{S}}$ & $0.2 \Omega$ \\
Equivalent load resistance, $\mathrm{R}_{\mathrm{L}}$ & $8.9 \Omega$ \\
Handshake communication signal matching capacitance, $\mathrm{C}_{1}$ & $1 \mathrm{nF}$ \\
High speed signal matching capacitance, $\mathrm{C}_{2}$ & $10 \mathrm{pF}$ \\
High speed signal matching capacitance, $\mathrm{C}_{3}$ & $1 \mathrm{nF}$ \\
\hline
\end{tabular}

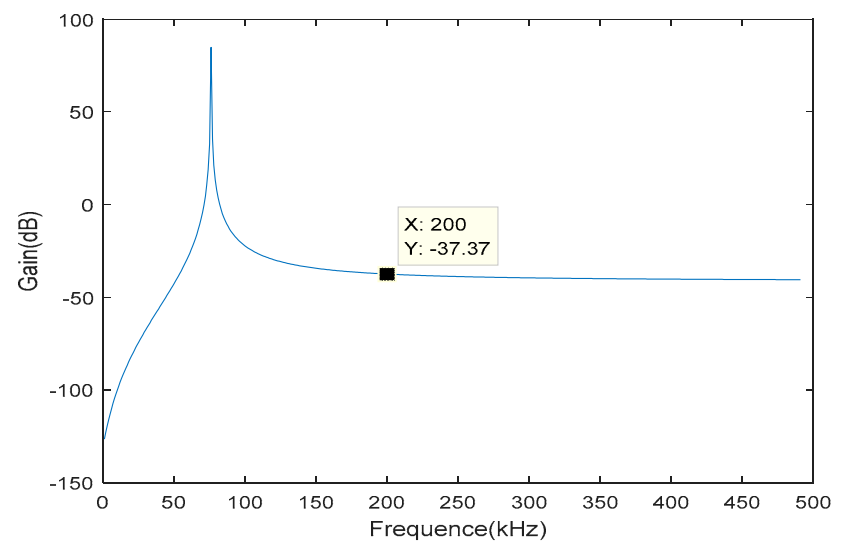

Figure 5. Control command interaction channel frequency response characteristic curve.

\subsection{Characteristics of High-Speed Signal Transmission Mode}

Through the SP high-frequency resonant network built on the coupled coil, high-speed signal transmission is achieved from robot fish to charging platform based on OFDM [18].

In the high-speed signal transmission mode, the equivalent circuit of the system is shown in Figure 6. 


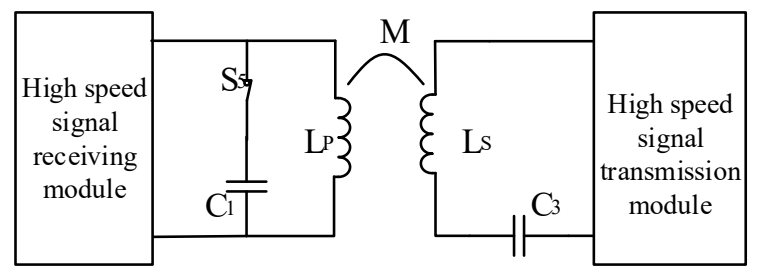

Figure 6. High-speed data transmission equivalent topology.

During data transmission, it is loaded into the SP resonance network by high-frequency modulation. In order to meet the high-speed data transmission requirements, the communication band of OFDM is selected in the frequency band of 2 to $30 \mathrm{MHz}$. The resonant frequency is set to $10 \mathrm{MHz}$, and the frequency response curve of the reverse data transmission channel is as shown in Figure 7.

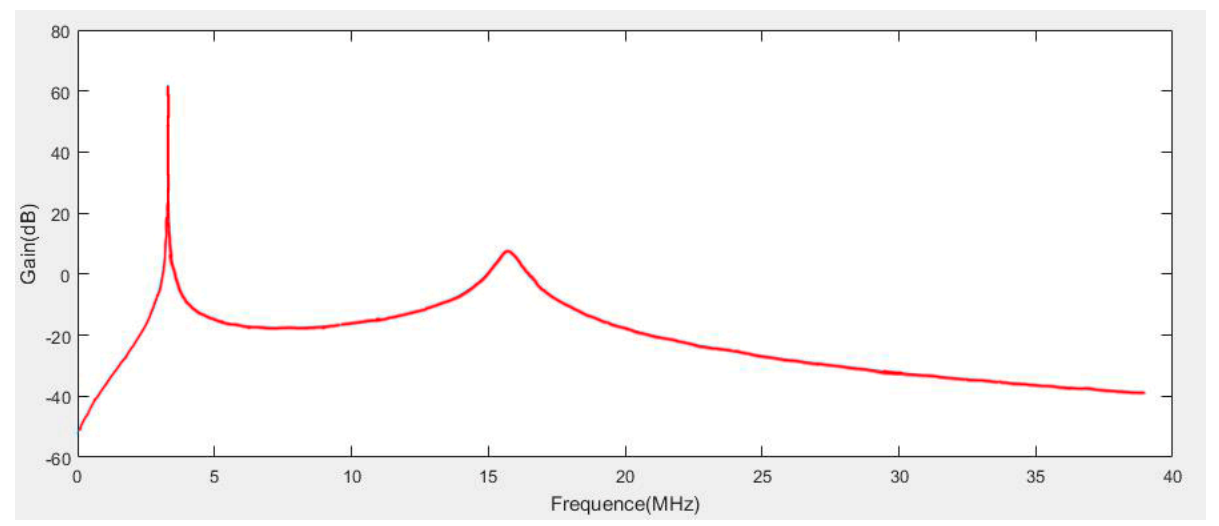

Figure 7. OFDM data transmission frequency response characteristic curve.

As shown in Figure 7, the signal transmission characteristic of the SP resonant network has a small attenuation in the frequency range of 2 to $28 \mathrm{MHz}$, and is suitable for OFDM carrier communication.

\section{Time-Sharing Multiplexing Strategy for Mode Switching}

When the inspection robot fish returns to the charging platform, in order to carry out efficient and reliable power supply and data transmission, the time-sharing multiplexing strategy for mode switching between the three working modes is shown in Figure 8.

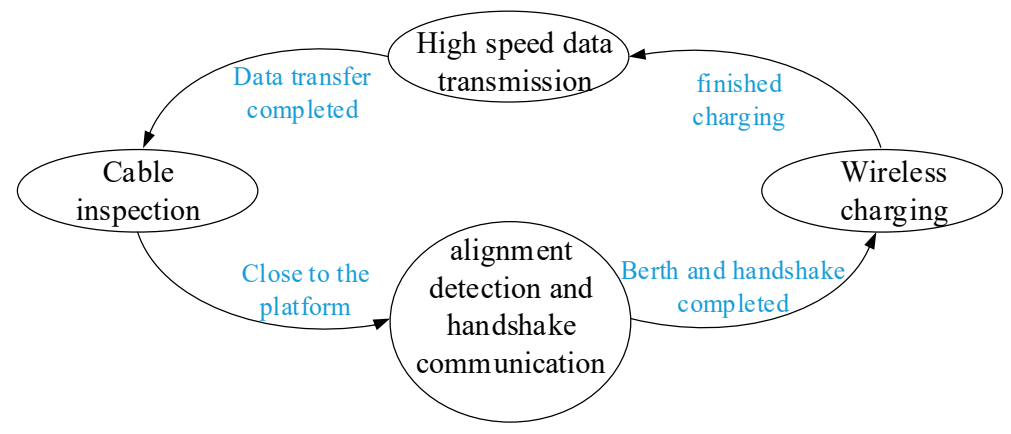

Figure 8. Mode switching diagram.

In the stage of standby, the charging platform periodically adjusts the handshake signal with the robot fish by inverter bridge in the modulation mode of AFK, and waits for the robot fish to respond. During the process of the robot fish approaching the platform, the robot fish reaches the top of the charging platform and performs a berth at the maximum point of the signal amplitude by detecting the 
amplitude of the received signal to determine the alignment between the platform and the robot fish. After the robot fish's berth is completed, the handshake signal sent by the platform is received and the feedback signal is modulated by the switching of switch $\mathrm{S}_{6}$. The charging platform demodulates the signal returned by the robot fish by detecting the change of the coil current, and enters the charging mode if the feedback signal is correct. During the charging process, the primary side collects current and voltage on the coil, and judges whether the charging is completed by calculating the equivalent impedance. After charging is completed, the robot fish enters the high-speed data transmission mode and transmits the submarine cable-inspection data packet. When all the data is sent, the charging platform checks whether the data is complete and sends a response. If the data is sent correctly, the robot fish enters the inspection state again, and the charging platform enters standby state.

The system workflow is shown in Figure 9.

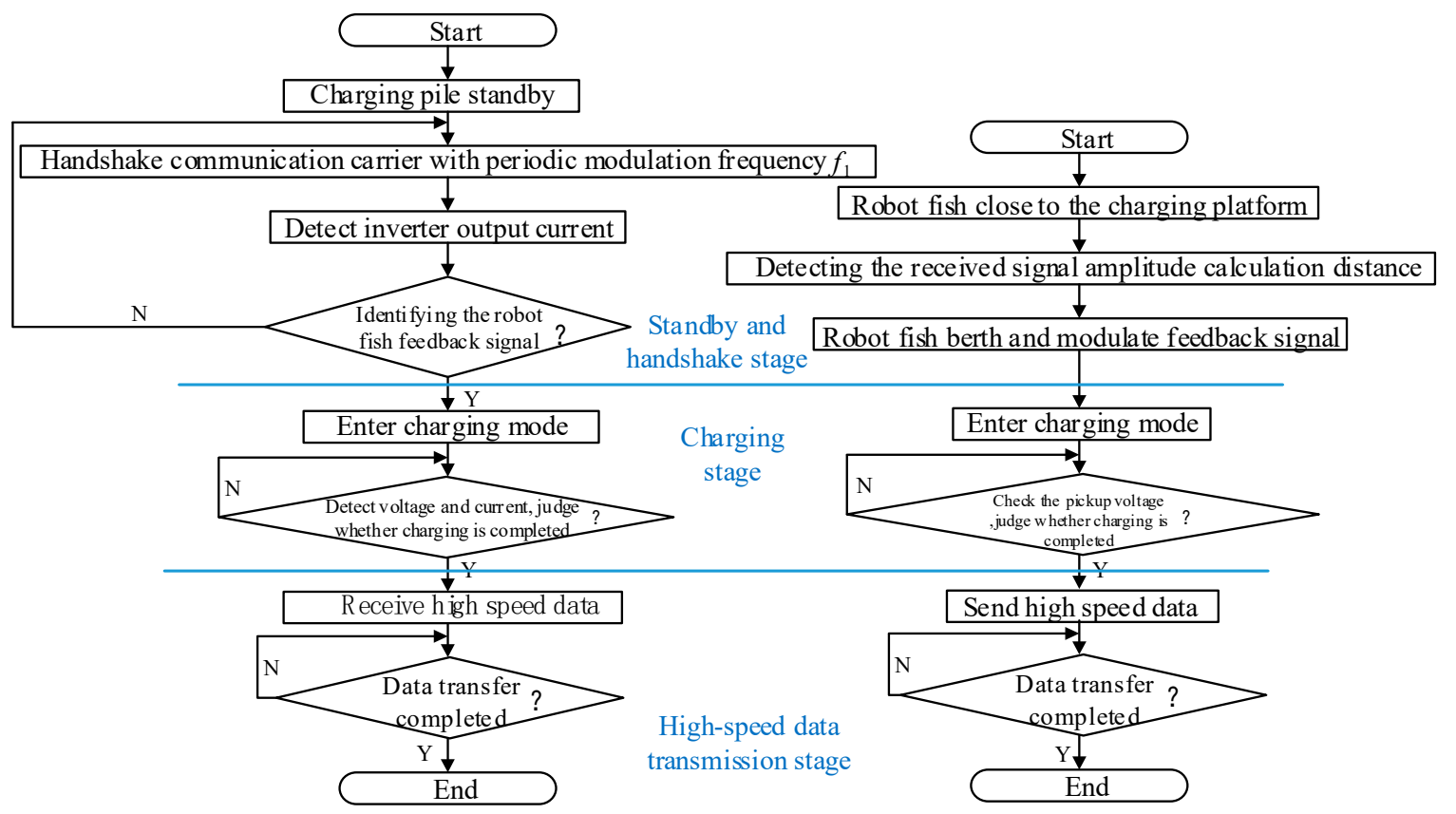

Figure 9. The flow chart of the system.

\section{Experimental Study}

In order to verify the feasibility of the wireless power and data transmission scheme of the shared channel mentioned above, the experimental device is built according to the system topology diagram shown in Figure 2, and the system parameters are designed as shown in Table 1 according to the parameter design method proposed in $[19,20]$. The power and data transmission performance and various indicators of the system are further verified by experiments.

The experimental prototype diagram is shown in Figure 10. The prototype includes power conversion, signal modulation and demodulation, coupling mechanism, and load composition. The power conversion part performs high-frequency inversion on the direct current and necessary overcurrent and overvoltage protection, realizes amplitude amplification by compensating the network, generates an alternating magnetic field on the resonant network, and transmits to the secondary side of the electric energy. The signal modulation and demodulation section complete the carrier signal modulation, the power amplification and the amplitude change of the carrier signal are shaped and restored to a digital signal to communicate with the host computer. The secondary side power conversion section provides rectification and voltage regulation functions to provide the DC power required for charging to the load. 


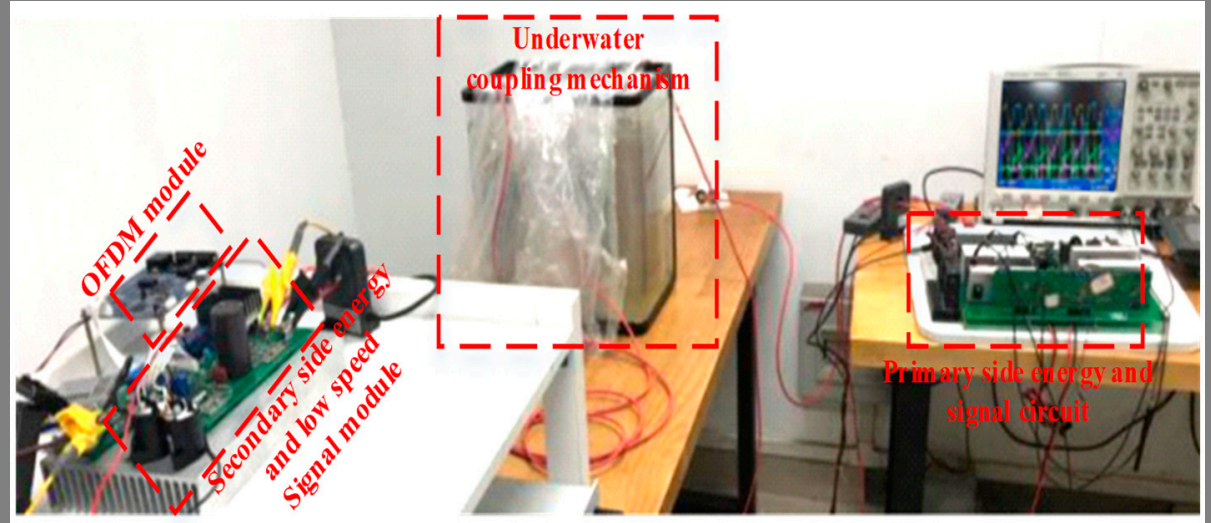

Figure 10. Experimental prototype diagram.

The experimental device handshake communication signal transmission part adopts half-duplex communication, and performs modulation and demodulation in ASK mode, the carrier frequency of the transmitted signal is $200 \mathrm{kHz}$. In order to make the working frequency and the signal carrier frequency of the power transmission mode far away from each other, the working frequency of the power transmission is designed to be a frequency of $85 \mathrm{kHz}$, which not only ensures the power transmission but also does not affect the communication effect. High-speed signal transmission uses a 2 to $28 \mathrm{MHz}$ carrier OFDM module for high-speed data transmission from the robotic fish to the charging pile. The energy and signal transmission experimental waveforms are shown in Figures 11-13, where Figure 11 shows the working waveform of the coil voltage and current at the time of power transmission. Figure 12 is a waveform diagram of a signal secondary side carrier signal and a demodulated signal during signal transmission. Figure 13 is a spectrum waveform obtained by fast Fourier transform of a picked-up signal in a high-speed signal transmission mode, which reflects the gain of each subcarrier in the OFDM modulation mode.

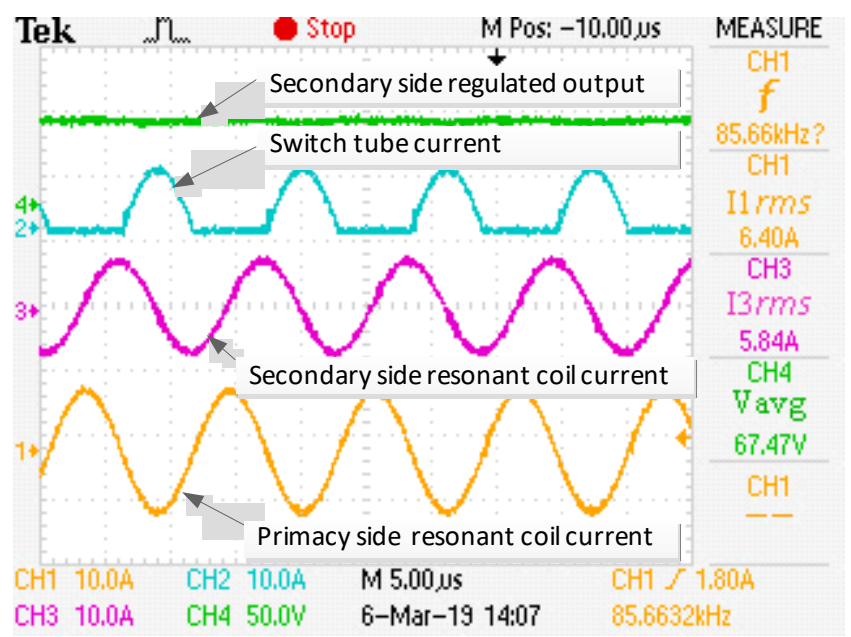

Figure 11. Power transmission waveform. 


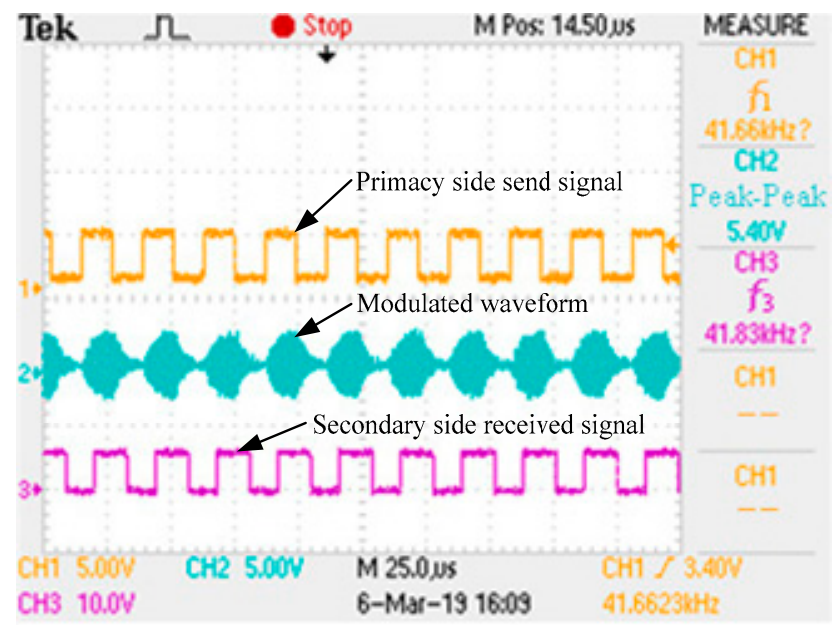

Figure 12. Low-speed signal transmission mode experiment waveform.

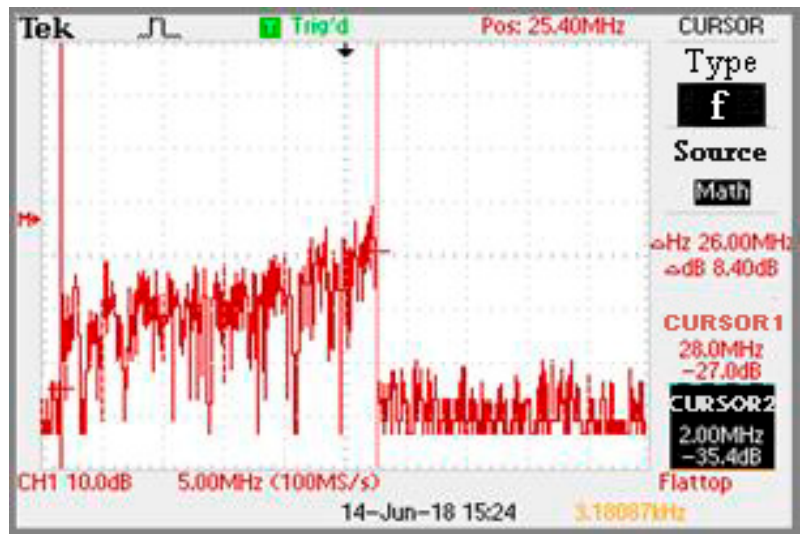

Figure 13. High-speed data transmission mode signal spectrum.

As it can be seen from Figure 11, since the power transmission portion operates at the resonance frequency point of the system, the system operates in a resonance state. At the same time, the experimental device was used to test different loads. The system works stably, the transmission power reaches $300 \mathrm{~W}$, and the efficiency is above $80 \%$, which satisfies the wireless charging demand of the submarine patrol robot fish.

From Figure 12, the ASK method can demodulate the digital signal very well. In the handshake communication signal transmission mode, the charging pile sends a control signal to the robot fish, the baud rate reaches $41 \mathrm{kbps}$, and the error rate of the system signal transmission is less than one ten thousandth. In practical applications, measures such as adding verification to signal transmission can ensure stable and reliable transmission of system signals, which can meet actual needs.

The high-speed signal transmission mode signal spectrum is shown in Figure 13. The data carrier has a frequency band of 2 to $28 \mathrm{MHz}$ and a frequency band of $26 \mathrm{MHz}$, which contains many subcarriers. The data transmission can be carried out steadily, and the transmission rate is maintained at $10 \mathrm{Mbps}$ or more, which satisfies the need for high-speed transmission of patrol data.

\section{Conclusions}

This article starts from the application requirements of the cable-inspection robotic fish wireless-charging system, based on the existing power and signal transmission methods, the two-way time-sharing transmission of power and signals in shared channels is studied. The electric energy coupling coils are used as the transmission channel of the signal, and the one-way transmission 
of electric energy and the bidirectional transmission of the signal are realized without interference between the electric energy and the signal. The transmission characteristics of the energy channel, the characteristics of alignment detection and low-speed handshake signal transmission, and the transmission characteristics of a high-speed signal are analyzed theoretically, and the corresponding channel switching control strategy is presented. The experimental device was built to verify the feasibility of the method, which gave an effective reference for the wireless charging, control and data transmission of the robot fish in the submarine cable-inspection system, and provided a solution for similar underwater robot wireless charging. This paper only explores the feasibility of the wireless energy and data transmission system and its time division multiplexing method; further exploration of output control and maximum efficiency tracking will be done soon.

Author Contributions: Supervision, methodology, writing-original draft preparation, G.C.; conceptualization, validation, investigation, resources, Y.S.; software, formal analysis, J.H.; B.Z.; resources, data curation, F.M.; supervision, funding acquisition, C.T.

Funding: National Natural Science Foundation of China(61573074); State Grid Corporation Science and Technology Project(5211DS17002F).

Conflicts of Interest: The authors declare no conflicts of interest.

\section{References}

1. Anqiang, L.; Yongqian, L.; Jing, L. Strain and Temperature Monitoring of $110 \mathrm{kV}$ Optical Fiber Composite Submarine Power Cable. High Volt. Eng. 2014, 40, 533-539.

2. Balasuriya, A.; Ura, T. Autonomous underwater vehicles for submarine cable inspection: Experimental results. IEEE 2001, 1, 377-382.

3. Zhang, B.; Shu, X.; Huang, R. The Development of Inductive and Resonant Wireless Power Transfer Technology. Trans. China Electrotech. Soc. 2017, 18, 9-23.

4. Li, S.; Liu, J.; Xu, H.; Zhao, H.; Wang, Y. Research status of autonomous underwater vehicles in China. Sci. Sin. Inform. 2018, 48, 1152-1164. [CrossRef]

5. Yuan, Z.; Yue, S.; Xin, D. Modeling and Analysis of Magnetic Resonance Wireless Power Transmission Systems. Trans. China Electrotech. Soc. 2012, 32, 155-160.

6. Lin, J.C. Space solar-power stations, wireless power transmissions, and biological implications. IEEE Trans. Microw. Mag. 2002, 3, 36-42. [CrossRef]

7. Shinohara, N.; Kawasaki, S. Recent Wireless Power Transmission technologies in Japan for space solar power station/satellite. In Proceedings of the Radio and Wireless Symposium, San Diego, CA, USA, 18-22 January 2009; pp. 13-15.

8. Bana, V.; Kerber, M.; Anderson, G.; Rockway, J.D.; Phipps, A. Underwater wireless power transfer for maritime applications. In Proceedings of the Wireless Power Transfer Conference, Boulder, CO, USA, 13-15 May 2015; pp. 1-4.

9. Zhu, C.; Yu, C.; Liu, K.; Ma, R. Research on the topology of wireless energy transfer device. In Proceedings of the IEEE Vehicle Power and Propulsion Conference, Harbin, China, 3-5 September 2008; Volume 9, pp. 3-5.

10. Mayordomo, I.; Dräger, T.; Spies, P.; Bernhard, J.; Pflaum, A. An overview of technical challenges and advances of inductive wireless power transmission. Proc. IEEE 2013, 101, 1302-1311. [CrossRef]

11. Su, Y.G.; Zhou, W.; Hu, A.P.; Tang, C.S.; Xie, S.Y.; Sun, Y. Full-duplex Communication on the Shared Channel of a Capacitively Coupled Power Transfer System. IEEE Trans. Power Electron. 2017, 32, 3229-3239. [CrossRef]

12. Sun, Y.; Yan, P.X.; Wang, Z.H.; Luan, Y.Y. The Parallel Transmission of Power and Data with the Shared Channel for an Inductive Power Transfer System. IEEE Trans. Power Electron. 2016, 31, 5495-5502. [CrossRef]

13. Yang, Q.; Li, Y.; Yin, J.; He, Y.; Xue, M. Wireless Synchronous Transmission of Power and Information Based on ASK in WPT via Coupled Magnetic Resonances. Trans. China Electrotech. Soc. 2017, 32, 153-161.

14. Han, J.W.; Kim, S.Y.; Kim, K.M.; Chun, S.Y.; Son, K. Design of OFDM system for high speed underwater communication. In Proceedings of the International Conference on Computational Science and Engineering, Vancouver, BC, Canada, 29-31 August 2009; pp. 988-992. 
15. Lucani, D.E.; Stojanovic, M.; Médard, M. On the relationship between transmission power and capacity of an underwater acoustic communication channel. In Proceedings of the OCEANS 2008-MTS/IEEE Kobe Techno-Ocean, Kobe, Japan, 8-11 April 2008.

16. Thrimawithana, D.J.; Madawala, U.K. A three-phase bi-directional IPT system for contactless charging of electric vehicles. In Proceedings of the IEEE International Symposium on Industrial Electronics, Gdansk, Poland, 27-30 June 2011; pp. 1957-1962.

17. Yue, S.; Zhibin, Z.; Zhihui, W.; Xin, D. A New Circuit of Soft-Switching Inductive Power Transfer System. Trans. China Electrotech. Soc. 2013, 288, 128-134.

18. Dai, L.; Wang, J.; Wang, Z.; Tsiaflakis, P.; Moonen, M. Spectrum- and Energy-Efficient OFDM Based on Simultaneous Multi-Channel Reconstruction. IEEE Trans. Signal Process. 2013, 61, 6047-6059. [CrossRef]

19. Dai, X.; Li, X.; Li, Y.; Hu, A.P. Impedance Matching Range Extension Method for Maximum Power Transfer Tracking in IPT System. IEEE Trans. Power Electron. 2017, 33, 4419-4428. [CrossRef]

20. Cho, S.Y.; Lee, I.O.; Moon, S.; Moon, G.W.; Kim, B.C.; Kim, K.Y. Series-series compensated wireless power transfer at two different resonant frequencies. In Proceedings of the Ecce Asia Downunder, Melbourne, Australia, 3-6 June 2013; pp. 1052-1058.

(C) 2019 by the authors. Licensee MDPI, Basel, Switzerland. This article is an open access article distributed under the terms and conditions of the Creative Commons Attribution (CC BY) license (http://creativecommons.org/licenses/by/4.0/). 